\title{
Efficacy of the Use of a Colorimetric Pupil Light Reflex Device in the Diagnosis of Fundus Disease or Optic Pathway Disease in Dogs
}

\author{
Kunihiko TERAKADO ${ }^{1) *}$, Takuya YOGO ${ }^{1)}$, Yoshinori NEZU ${ }^{1)}$, Yasuji HARADA ${ }^{1)}$, Yasushi HARA ${ }^{1)}$ and \\ Masahiro TAGAWA ${ }^{1)}$ \\ ${ }^{1)}$ Department of Veterinary Surgery, Nippon Veterinary and Life Science University, 1-7-1 Kyonancho, Musashino, Tokyo 180-8602, \\ Japan
}

(Received 15 August 2012/Accepted 4 June 2013/Published online in J-STAGE 18 June 2013)

ABSTRACT. The aim of this study was to determine the efficacy of a colorimetric pupil light reflex (PLR) device (Melan-100 ${ }^{\circledR}$, U.S.A.) in dogs with sudden acquired retinal degeneration syndrome (SARDS; 16 cases), progressive retinal atrophy (PRA; 10 cases) and optic pathway disease (6 cases). The colorimetric device detected PLR abnormality in 32, 16 and 9 eyes with SARDS, PRA and optic pathway disease, respectively, whereas white light detected PLR abnormality in 18,11 and 9 eyes with SARDS, PRA and optic pathway disease, respectively. SARDS dogs displayed miosis, while optic pathway disease dogs displayed mydriasis in a blue light examination. Thus, colorimetric PLR may be a useful method for determining whether electroretinography (ERG) or magnetic resonance imaging (MRI) should be performed for dogs with acute blindness.

KEY WORDS: canine, colorimetric pupil light reflex, neurological disease, progressive retinal atrophy, sudden acquired retinal degeneration syndrome.

doi: 10.1292/jvms.12-0363; J. Vet. Med. Sci. 75(11): 1491-1495, 2013

Ophthalmic disorders causing blindness include sudden acquired retinal degeneration syndrome (SARDS), progressive retinal atrophy (PRA) or optic pathway disease.

Each of these disorders is described below. The first one, SARDS, is an idiopathic disorder that results in rapid and irreversible vision loss in affected dogs and is characterized by the sudden onset of vision loss due to the complete loss of photoreceptor activity. This vision loss is examined by non-recordable electroretinography (ERG), and SARDS is caused by acute damage to the outer photoreceptor segments $[12,17]$. Dogs with SARDS are typically mydriatic at rest with a diminished pupil light reflex (PLR), and the results of a funduscopic examination are unremarkable at the early stages of the disease $[6,18]$. For diagnosis of SARDS, a triad of findings is required: a history of sudden vision loss, normal results of a funduscopic examination and extinguished retinal activity as determined by ERG [1].

The second disease is PRA. By definition, PRA is an inherited, bilateral, progressive degeneration of the retina that eventually results in blindness [1]. In general, PRA initially manifests as a disease of the photoreceptors and eventually involves all the retinal layers. Dogs with PRA are also mydriatic at rest with diminished PLR. On ophthalmoscopic examination, fundus lesions appear with increased tapetal reflectivity, and many breeds may develop fan-shaped striations of altered tapetal reflectivity that converge at the disc. Concurrently, retinal blood vessels become attenuated. Ab-

*Correspondence to: Terakado, K., Department of Veterinary Surgery, Nippon Veterinary and Life Science University, 1-7-1 Kyonancho, Musashino, Tokyo 180-8602, Japan.

e-mail: terakado.vet@gmail.com

(C)2013 The Japanese Society of Veterinary Science normalities in ERG amplitude and latency will occur much earlier than ophthalmoscopic changes. Late in the course of the disease, the ERG is non-recordable. The diagnosis of PRA requires night blindness or day blindness, characteristic ophthalmoscopic changes, abnormalities in ERG and genetic testing for abnormalities.

The third disease is optic pathway disease. Optic neuritis (ON) may be unilateral or bilateral and can involve the optic disc or be confined to the retrobulbar nerve. Dogs with bilateral involvement are blind with dilated pupils that typically respond very sluggishly and incompletely to light. The fundus may be normal if the condition is completely retrobulbar, but the typical appearance is congestion and a swollen optic disc with deviation of the retinal blood vessels. Central blindness resulting in progressive vision loss has been reported in association with hydrocephalus, neoplasia of the central nervous system and granulomatous meningoencephalomyelitis (GME) [5, 16]. Intracranial tumors compressing the optic chiasm result in total vision loss with complete or near-complete loss of PLR [3]. Optic pathway disease resulting in acute blindness is characterized by a normal ERG, thereby differentiating it from SARDS. However, additional diagnostic tests, such as magnetic resonance imaging (MRI) and cerebrospinal fluid analysis, are necessary to obtain a definitive diagnosis.

The technique of PLR detects lesions on the retina and optic nerve, and blind dogs with retinal lesions retain PLR [7]. For example, blind dogs with non-recordable ERG, such as those with SARDS, could retain PLR. A colorimetric PLR device (Melan-100 ${ }^{\circledR}$; BioMed Vision Technologies, Ames IA, U.S.A.) is used accessorily for diagnosis of some fundus disease in dogs. This colorimetric PLR device is based on the principle that retinal ganglion cells irradiated with strong blue light induce PLR [7]. Light-sensitive cells, such as pho- 
Table1. Clinical data and diagnosis of 32 fundus disease dogs

\begin{tabular}{|c|c|c|c|c|c|c|c|c|c|}
\hline No. & Breed & Age (year) & Sex & $\mathrm{OD} / \mathrm{OS}$ & Menace & White light & Red light & Blue light & Diagnosis \\
\hline \multirow[b]{2}{*}{1} & \multirow[b]{2}{*}{ Dachshund } & \multirow{2}{*}{10} & \multirow{2}{*}{ SF } & OD & - & \pm & - & \pm & \multirow{2}{*}{ SARDS } \\
\hline & & & & OS & - & \pm & - & \pm & \\
\hline \multirow[b]{2}{*}{2} & \multirow{2}{*}{ Shetland sheepdog } & \multirow[b]{2}{*}{8} & \multirow{2}{*}{ NM } & OD & - & \pm & - & \pm & \multirow[b]{2}{*}{ SARDS } \\
\hline & & & & OS & - & \pm & - & \pm & \\
\hline \multirow{2}{*}{3} & \multirow{2}{*}{ Miniature schnauzer } & \multirow[b]{2}{*}{7} & \multirow[b]{2}{*}{$\mathrm{F}$} & OD & - & + & \pm & + & \multirow{2}{*}{ SARDS } \\
\hline & & & & OS & - & + & \pm & + & \\
\hline \multirow[b]{2}{*}{4} & \multirow[b]{2}{*}{ Dachshund } & \multirow[b]{2}{*}{6} & \multirow{2}{*}{ NM } & OD & - & + & \pm & + & \\
\hline & & & & OS & - & + & \pm & + & SARDS \\
\hline & & & & OD & - & - & - & + & \\
\hline 5 & French bulldog & 7 & NM & OS & - & - & - & + & SARDS \\
\hline & & & & OD & - & - & - & + & \\
\hline 6 & Mix & 13 & $\mathrm{SF}$ & OS & - & - & - & + & SARDS \\
\hline & & & & OD & - & + & - & + & \\
\hline 7 & Mix & 10 & $\mathrm{SF}$ & OS & - & + & - & + & SARDS \\
\hline & & & & OD & - & \pm & - & + & \\
\hline 8 & Dachshund & 10 & $\mathrm{SF}$ & OS & - & \pm & - & + & SARDS \\
\hline 9 & Dachshund & & & OD & - & + & - & + & \\
\hline 9 & Dachshund & 7 & M & OS & - & + & - & + & SARDS \\
\hline & & & & OD & - & - & - & + & \\
\hline 10 & Dachshund & 9 & NM & OS & - & - & - & + & SARDS \\
\hline & & & & OD & - & - & - & + & \\
\hline 11 & Miniature schnauzer & 10 & NM & OS & - & - & - & + & SARDS \\
\hline 12 & Dochchund & & & OD & - & \pm & - & + & \\
\hline 12 & Dachshund & 11 & M & OS & - & - & - & + & SARDS \\
\hline & & & & OD & - & \pm & - & + & \\
\hline 13 & Lhasa apso & 10 & $\mathrm{~F}$ & OS & - & \pm & - & + & SARDS \\
\hline & & & & OD & - & \pm & - & + & \\
\hline 14 & Minıature schnauzer & 8 & $\mathrm{SF}$ & OS & - & \pm & - & + & SARDS \\
\hline & & & & OD & - & - & - & + & \\
\hline 15 & Cairn terrier & 9 & NM & OS & - & - & - & + & SARDS \\
\hline 16 & & 5 & $F_{2} \rightarrow$ & OD & - & \pm & - & + & \\
\hline 16 & Dachshund & 5 & $\mathrm{~F}$ & OS & - & \pm & - & + & SARDS \\
\hline 17 & & & & OD & - & - & - & + & \\
\hline 17 & Chihuahua & 7 & $\mathrm{~F}$ & OS & - & - & - & + & PRA \\
\hline & & & & OD & \pm & \pm & - & + & \\
\hline 18 & Dachshund & 11 & $\mathrm{~F}$ & OS & + & \pm & - & + & PRA \\
\hline & & & & OD & - & + & \pm & + & PDA \\
\hline 19 & Dachshund & 1 & M & OS & - & + & \pm & + & PRA \\
\hline 20 & & & & OD & + & + & + & + & \\
\hline 20 & Dachshund & 1 & NM & OS & + & + & + & + & PRA \\
\hline 21 & Doshshund & & & OD & - & - & - & + & \\
\hline 21 & Dachshund & 2 & SF & OS & - & - & - & + & PRA \\
\hline 22 & Dochsund & 4 & $M$ & OD & - & - & - & \pm & PPA \\
\hline 22 & Dacnsnund & 4 & $\mathrm{M}$ & OS & - & - & - & \pm & PRA \\
\hline 23 & Miniature schnauzer & 9 & $\mathrm{SF}$ & OS & - & - & - & - & PRA \\
\hline 24 & Mix & 10 & M & OS & - & + & _- & + & PRA \\
\hline 25 & Donblbund & 0 & $\mathrm{~S}$ & OD & - & \pm & - & + & DD \\
\hline 25 & Dachshund & 9 & $\mathrm{SF}$ & OS & - & - & - & + & PRA \\
\hline & & & & OD & - & + & - & + & \\
\hline 26 & Miniature schnauzer & 13 & $\mathrm{NM}$ & OS & - & + & - & + & PRA \\
\hline
\end{tabular}




\begin{tabular}{|c|c|c|c|c|c|c|c|c|c|}
\hline 27 & Cavalier King Charles spaniel & 5 & SF & $\begin{array}{l}\text { OD } \\
\text { OS }\end{array}$ & $\begin{array}{l}- \\
-\end{array}$ & $\begin{array}{l}- \\
-\end{array}$ & $\begin{array}{l}- \\
-\end{array}$ & $\begin{array}{l}- \\
-\end{array}$ & ON \\
\hline 28 & French bulldog & 2 & $\mathrm{~F}$ & OS & - & - & - & - & ON \\
\hline 29 & Pembroke Welsh corgi & 11 & $\mathrm{~F}$ & $\begin{array}{l}\text { OD } \\
\text { OS }\end{array}$ & $\begin{array}{l}- \\
-\end{array}$ & $\begin{array}{l}- \\
-\end{array}$ & $\begin{array}{l}- \\
-\end{array}$ & $\begin{array}{l}- \\
-\end{array}$ & Pituitary tumor \\
\hline 30 & Dachshund & 8 & $\mathrm{~F}$ & $\begin{array}{l}\text { OD } \\
\text { OS }\end{array}$ & $\begin{array}{l}- \\
-\end{array}$ & $\begin{array}{l}- \\
-\end{array}$ & $\begin{array}{l}- \\
-\end{array}$ & $\begin{array}{l}- \\
-\end{array}$ & GME \\
\hline 31 & Beagle & 13 & M & OD & - & - & - & - & Meningioma \\
\hline 32 & Dachshund & 11 & $\mathrm{~F}$ & OS & - & - & - & - & Meningioma \\
\hline
\end{tabular}

M: Intact male, NM: Neutered male, F: Intact female, SF: Spayed female. OD: Oculus Dexter, OS: Oculus sinister. The Fundus could not be observed, because of cataract in No. 23 (OD), No. 24 (OD) and No. 28 (OD). No. 31 (OS) and No. 32 (OD) were normal.

toreceptors and parts of retinal ganglion cells, exist in the retina. These retinal ganglion cells contain a photopigment named melanopsin, and melanopsin-containing retinal ganglion cells irradiated with light at a wavelength of 420-440 nm induce PLR $[4,10,15]$. Therefore, red $(630 \mathrm{~nm})$ and blue $(480 \mathrm{~nm})$ light-emitting diodes (LEDs) with an intensity of $200 \mathrm{kcd} / \mathrm{m}^{2}$ are used as stimulation. Normal PLR is induced by photoreceptors and melanopsin-containing retinal ganglion cells $[8,9,11]$. A red LED causes a reaction only in the photoreceptor, but a blue LED activates both the photoreceptor and the melanopsin-containing retinal ganglion cells.

The usefulness of colorimetric PLR for SARDS dogs has been previously reported [7]. However, the efficacy of the use of colorimetric PLR for diagnosing PRA and optic pathway disease has not been compared. Additionally, the efficacy of the use of colorimetric PLR for diagnosis of blindness in dogs with SARDS and those with optic pathway disease has also not been compared. Thus, we compared the efficacy of the use of colorimetric PLR for healthy dogs with SARDS, PRA and optic pathway disease.

We reviewed cases of SARDS, PRA and optic pathway disease in 33 dogs (61 eyes) in which a Melan-100 was used at the Animal Medical Center of Nippon Veterinary and Life Science University between November 2009 and November 2011. Medical history and menace response were recorded, and measurement of PLR and intraocular pressure, slit lamp examination, funduscopic examination and ERG were conducted for all dogs. The criteria for SARDS are a history of sudden vision loss, normal results of a funduscopic examination and non-recordable ERG. The criteria for PRA are a history of night blindness or day blindness, characteristic ophthalmoscopic changes and subnormal or non-recordable ERG. The criteria for optic pathway disease are a history of vision defect, fundus photograph, normal ERG results and abnormal MRI findings within the optic pathway, such as in the optic nerve or optic chiasm. The ERG pro,tocol was based on the protocol used in a previous study [14]. In suspected cases of SARDS and optic pathway disease, first, retinal function was examined in ambient light using a standard flash (SF). An SF was used with a white-flash stimulus intensity of $2-3 \mathrm{~cd} / \mathrm{m}^{2} / \mathrm{sec}$. Second, the light was turned off, and retinal function was tested within the first few min of dark adaptation using the SF intensity. Finally, retinal function was tested again after 5 min of dark adaptation by using the SF intensity. In suspected cases of PRA, first, the rod function was evaluated by the dynamic process of dark adaptation every $4 \mathrm{~min}$ (at 1, 4, 8, 12, 16 and $20 \mathrm{~min}$ ). Intensity of the white flash stimulus was $2 \log$ units below that of the SF. Second, the mixed rod and cone response was tested using the SF. Third, cone function was tested using the SF after 10 min of light adaptation. Finally, cone flicker response was evaluated at a frequency of $30 \mathrm{~Hz}$ using SF intensity. In the control group, 10 healthy experimental dogs (20 eyes) underwent ophthalmological examination (menace response, PLR, measurement of intraocular pressure, slit lamp examination, funduscopic examination and ERG). Dogs in which the fundus was not observed because of a cataract were excluded from this study.

One examiner assessed the PLR using a colorimetric device and white light, while the dogs were kept in a sitting position by animal health technicians. We conformed to the Melan-100 instructions regarding its use and evaluation. In a dark examination room, we waited for $30 \mathrm{sec}$ in order to achieve dark adaptation. Then, we illuminated the right eye using the red light from the front and at a distance of 1 inch for not more than $10 \mathrm{sec}$ and observed changes in the pupil diameter using a scale. A pupil diameter of less than 4 mm was described as + , decreased PLR was described as \pm , and absence of PLR was described as -. If complete pupil constriction was achieved in less than $10 \mathrm{sec}$, the light source could be turned off immediately. We then waited for $30 \mathrm{sec}$ so that the eye could readapt, and then, the left eye was illuminated with red light using the same method. Using the same procedure, both eyes were examined using blue light after $30 \mathrm{sec}$.

The 33 dogs with fundus or optic pathway disease (61 eyes) were evaluated for white light PLR by using a Finoff transilluminator (Welch Allyn Inc., Skaneateles, NY, U.S.A.) and for red light and blue light PLR by using a Melan-100 for each disease (SARDS, PRA and optic pathway disease). 
White light PLR was performed using the same procedure as used for colorimetric PLR. The sensitivity and specificity of the Melan-100 were calculated using dogs with fundus disease and healthy dogs. Statistical analysis of sensitivity, specificity, false-positive rate, false-negative rate, positive predictive value and negative predictive value were performed using the SPSS 16.0 software package (SPSS Inc., Chicago, IL, U.S.A.).

The clinical data from dogs with fundus or optic pathway disease are shown in Table 1. Miosis was observed in all control dogs with white, red and blue light PLR.

The sensitivity was $96.2 \%$; the specificity was $100 \%$; the false-positive rate was $0 \%$; the false-negative rate was $3.8 \%$; the positive predictive value was $100 \%$; and the negative predictive value was $90.9 \%$.

Of the 33 dogs included in the study, 16 (32 eyes) were diagnosed with SARDS. Breeds that comprised $10 \%$ or more of the population of SARDS dogs included the dachshund (44\%), miniature schnauzer (19\%) and mixed breeds $(13 \%)$. The ages of the dogs at the time of diagnosis ranged from 5.5 to 13 years with a median age of 9.4 years at the time of diagnosis. Five were spayed females, 3 were intact females, 6 were neutered males, and 2 were intact males. The instructions for the Melan-100 state that SARDS dogs show no PLR response to red light and miosis to blue light. The results of 24 eyes of SARDS dogs corresponded to the device's instructions, but 8 eyes did not. Four eyes showed weak miosis on red light exposure and miosis on blue light exposure. The other 4 eyes displayed mydriasis on red light exposure and weak miosis on blue light exposure. The device detected PLR abnormality in all 32 eyes of SARDS dogs, but only 24 eyes (75\%) with PLR abnormalities were detected on white light exposure.

Of the 33 dogs included in the study, 10 dogs (18 eyes) were diagnosed with PRA. Breeds that comprised $10 \%$ or more of the population of PRA dogs included the dachshund $(60 \%)$, miniature schnauzer $(20 \%)$, Chihuahua $(10 \%)$ and mixed breeds $(10 \%)$. The ages of the dogs at the time of diagnosis ranged from 1 year to 13.9 years with a median age of 8.3 years at the time of diagnosis. Three were spayed females, 2 were intact females, 2 were neutered males, and 3 were intact males. The instructions for the Melan-100 state that PRA dogs show a decreased PLR response to red light and miosis to blue light. The reactions from 2 eyes of PRA dogs corresponded to the instructions, but 16 eyes did not. Two eyes displayed miosis on exposure to red and blue light. Eleven eyes displayed mydriasis on exposure to red light, and miosis on exposure to blue light. Three eyes displayed mydriasis on exposure to red light and weak miosis or mydriasis on exposure to blue light. In the 18 eyes with PRA, PLR abnormality was detected in 16 eyes $(88.9 \%)$ by the Melan-100 and in 11 eyes (61.1\%) on white light exposure.

Of the 33 dogs included in the study, 6 dogs (9 eyes) were diagnosed with optic pathway disease. The only breed that comprised $10 \%$ or more of the population of optic pathway disease dogs was the dachshund (33\%). The ages of the dogs at the time of diagnosis ranged from 2 year to 13.1 years with a median age of 8.3 years at the time of diagnosis. One was a spayed female, 4 were intact females, and 1 was an intact males. Two dogs had ON, 1 had a pituitary tumor, 1 had GME, and 2 had meningioma. The instructions for the Melan-100 state that dogs with optic pathway disease show no PLR response to red or blue light exposure. Our results corresponded to these instructions. All eyes with optic pathway disease had PLR abnormality as determined by both the Melan-100 and white light exposure.

Use of a Melan-100 as a screening tool might aid in the detection of fundus abnormalities, because of its high sensitivity $(96.2 \%)$ and low false-negative rate $(0 \%)$. The device is useful as a diagnostic tool at clinical sites, because of its higher positive predictive value $(100 \%)$ and negative predictive value $(90.9 \%)$. The usefulness of colorimetric PLR for SARDS dogs has been reported, and colorimetric PLR is useful as a diagnostic aid for PRA and optic pathway disease. Although a colorimetric PLR device is useful for screening, it is risky to diagnose these abnormalities with this method alone. It is necessary to diagnose SARDS, PRA and optic pathway disease via funduscopic examination, ERG and MRI.

The results for 24 eyes of SARDS dogs corresponded to the device's instructions (75\%). In the 8 eyes whose reactions differed from the instructions, 4 eyes showed weak miosis when exposed to red light, but the ERG was nonrecordable. The other 4 eyes displayed weak miosis on blue light exposure. This might indicate an extension of retinal degeneration for the retinal ganglion cells. All 32 eyes with SARDS had PLR abnormalities detected by the colorimetric PLR device, while 24 eyes (75\%) had PLR abnormalities detected by white light exposure. In a previous report, the proportion of PLR abnormalities detected by white light exposure was $63 \%$ in SARDS dogs [13]. These results suggest that the colorimetric PLR device is more useful than observing white light PLR in the detection of dogs with SARDS.

The results for 2 eyes of PRA dogs corresponded to the instructions $(11.1 \%)$. In the 16 eyes whose results differed from the instructions, 11 displayed mydriasis on exposure to red light and miosis on exposure to blue light. Three eyes displayed mydriasis on exposure to red light and weak miosis or mydriasis on exposure to blue light. Due to progression of atrophy, photoreceptors and retinal ganglion cells might become damaged and severely degenerated. The other 2 eyes displayed normal reactions to red and blue light exposure; this dog with PRA showed normal reactions to red and blue light exposure and presented a normal fundus, but had a history of night blindness and showed subnormal ERG results. Atrophy of the retina may not be detected by the colorimetric PLR device in the early stages of PRA. In the 18 eyes diagnosed with PRA, 16 eyes (88.9\%) were detected with PLR abnormality by the colorimetric PLR device, whereas 11 eyes (61.1\%) were detected with PLR abnormality by white light exposure. These results also suggest that the colorimetric PLR device is more useful than observing white light PLR in the detection of PRA dogs.

The results for the dogs diagnosed with optic pathway disease corresponded to the instructions in all eyes. Both colorimetric and white light PLR detected abnormalities in 
optic pathway disease dogs.

White light contains red, green and blue light. Illumination with blue light revealed miosis in 11 eyes of SARDS dogs and 7 eyes of PRA dogs, but white light did not do so. The melanopsin-containing retinal ganglion cells responded more strongly when strong light was applied [2]. The blue light of the Melan-100 has a high intensity, but the white light of the Finoff transilluminator has a low intensity. This raises the possibility that a contracted pupil was observed with blue light, and it was not observed with white light.

The colorimetric PLR results of SARDS dogs were very similar to PRA dogs. Therefore, it is difficult to distinguish SARDS and PRA using a colorimetric PLR device. When comparing the colorimetric PLR dogs with PRA and optic pathway disease, PRA tended to display miosis on exposure to blue light, while dogs with optic pathway disease displayed mydriasis on exposure to blue light. However, there is a possibility that miosis on exposure to blue light is not displayed due to the progress of PRA. Therefore, it might require attention to distinguish PRA and optic pathway disease using colorimetric PLR device. When comparing the colorimetric PLR results of SARDS and optic pathway disease dogs, SARDS dogs displayed miosis on exposure to blue light, while optic pathway disease dogs displayed mydriasis on exposure to blue light.

Our hypothesis was that PLR observed using the colorimetric PLR device could distinguish between SARDS and optic pathway disease before funduscopic examination and ERG could. In dogs with acute blindness, dogs with SARDS retain blue light PLR, but dogs with optic pathway disease do not. Conducting ERG or MRI for dogs with acute blindness requires that the dogs be immobilized by sedation or general anesthesia. However, colorimetric PLR can be performed without sedation or general anesthesia. The results of colorimetric PLR may provide a useful indication of whether ERG or MRI should be performed for dogs with acute blindness. This study included only 6 dogs with optic pathway disease; a higher number of dogs with optic pathway disease may have increased the reliability of the study.

\section{REFERENCES}

1. Charles, L. M. 2010. Vitreous and ocular fundus. pp. 422-431. In: Ophthalmic Disease in Veterinary Medicine (Charles, L. M. ed.), Manson Publishing, London.

2. Dacey, D. M., Liao, H. W., Peterson, B. B., Robinson, F. R., Smith, V. C., Pokorny, J., Yau, K. W. and Gamlin, P. D. 2005. Melanopsin-expressing ganglion cells in primate retina signal colour and irradiance and project to the LGN. Nature 433: 749-754. [Medline] [CrossRef]

3. Davidson, M. G., Nasisse, M. P., Breitschwerdt, E. B., Thrall, D. E., Page, R. L., Jamieson, V. E. and English, R. V. 1991. Acute blindness associated with intracranial tumors in dogs and cats: eight cases (1984-1989). J. Am. Vet. Med. Assoc. 199: 755-758. [Medline]
4. Foster, R. G. 2005. Neurobiology: bright blue times. Nature 433: 698-699. [Medline] [CrossRef]

5. Fischer, C. A. and Jones, G. T. 1972. Optic neuritis in dogs. J. Am. Vet. Med. Assoc. 160: 68-79. [Medline]

6. Gilmour, M. A., Cardenas, M. R., Blaik, M. A., Bahr, R. J. and McGinnis, J. F. 2006. Evaluation of a comparative pathogenesis between cancer-associated retinopathy in humans and sudden acquired retinal degeneration syndrome in dogs via diagnostic imaging and western blot analysis. Am. J. Vet. Res. 67: 877-881. [Medline] [CrossRef]

7. Grozdanic, S. D., Matic, M., Sakaguchi, D. S. and Kardon, R. H. 2007. Evaluation of retinal status using chromatic pupil light reflex activity in healthy and diseased canine eyes. Invest. Ophthalmol. Vis. Sci. 48: 5178-5183. [Medline] [CrossRef]

8. Güler, A. D., Ecker, J. L., Lall, G. S., Haq, S., Altimus, C. M., Liao, H. W., Barnard, A. R., Cahill, H., Badea, T. C., Zhao, H., Hankins, M. W., Berson, D. M., Lucas, R. J., Yau, K. W. and Hattar, S. 2008. Melanopsin cells are the principal conduits for rodcone input to non-image-forming vision. Nature 453: 102-105. [Medline] [CrossRef]

9. Hatori, M., Le, H., Vollmers, C., Keding, S. R., Tanaka, N., Buch, T., Waisman, A., Schmedt, C., Jegla, T. and Panda, S. 2008. Inducible ablation of melanopsin-expressing retinal ganglion cells reveals their central role in non-image forming visual responses. PLoS One 3: e2451. [Medline] [CrossRef]

10. Hattar, S., Liao, H. W., Takao, M., Berson, D. M. and Yau, K. W. 2002. Melanopsin-containing retinal ganglion cells: architecture, projections, and intrinsic photosensitivity. Science $\mathbf{2 9 5}$ 1065-1070. [Medline] [CrossRef]

11. Martin, C. L. 2001. Evaluation of patients with decreased vision or blindness. Clin. Tech. Small Anim. Pract. 16: 62-70. [Medline] [CrossRef]

12. Miller, P. E., Galbreath, E. J., Kehren, J. C., Steinberg, H. and Dubielzig, R. R. 1998. Photoreceptor cell death by apoptosis in dogs with sudden acquired retinal degeneration syndrome. $\mathrm{Am}$. J. Vet. Res. 59: 149-152. [Medline]

13. Montgomery, K. W., van der Woerdt, A. and Cottrill, N. B. 2008. Acute blindness in dogs: sudden acquired retinal degeneration syndrome versus neurological disease (140 cases, 2000-2006). Vet. Ophthalmol. 11: 314-320. [Medline] [CrossRef]

14. Narfström, K., Ekesten, B., Rosolen, S. G., Spiess, B. M., Percicot, C. L. and Ofri, R. 2002. Guidelines for clinical electroretinography in the dog. Doc. Ophthalmol. 105: 83-92. [Medline] [CrossRef]

15. Newman, L. A., Walker, M. T., Brown, R. L., Cronin, T. W. and Robinson, P. R. 2003. Melanopsin forms a functional shortwavelength photopigment. Biochemistry 42: 12734-12738. [Medline] [CrossRef]

16. Nuhsbaum, M. T., Powell, C. C., Gionfriddo, J. R. and Cuddon, P. A. 2002. Treatment of granulomatous meningoencephalomyelitis in a dog. Vet. Ophthalmol. 5: 29-33. [Medline] [CrossRef]

17. O'Toole, D., Roberts, S. and Nunamaker, C. 1992. Sudden acquired retinal degeneration ('silent retina syndrome') in two dogs. Vet. Rec. 130: 157-161. [Medline] [CrossRef]

18. van der Woerdt, A., Nasisse, M. P. and Davidson, M. G. 1991. Sudden acquired retinal degeneration in the dog: clinical and laboratory findings in 36 cases. Prog. Vet. Comp. Ophthalmol. 1: $11-18$. 